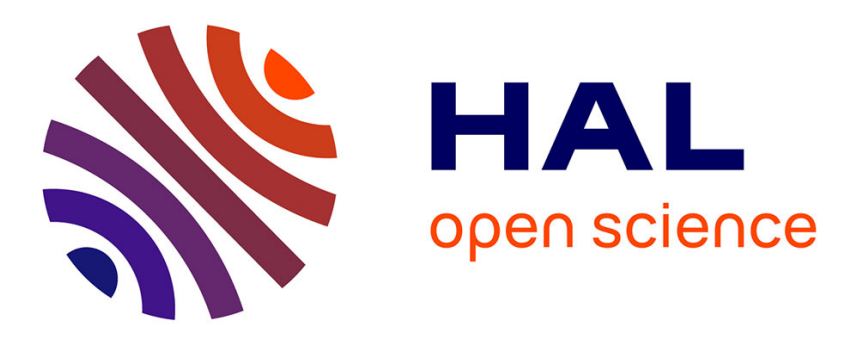

\title{
Application of the method of manufactured solutions to the verification of a pressure-based finite-volume numerical scheme
}

João Marcelo Vedovoto, Aristeu Da Silveira Neto, Arnaud Mura, Luis Fernando Figueira da Silva

\section{To cite this version:}

João Marcelo Vedovoto, Aristeu Da Silveira Neto, Arnaud Mura, Luis Fernando Figueira da Silva. Application of the method of manufactured solutions to the verification of a pressure-based finite-volume numerical scheme. Computers and Fluids, 2011, 51 (1), pp.85-99. 10.1016/j.compfluid.2011.07.014. hal-03313644

\section{HAL Id: hal-03313644 \\ https://hal.science/hal-03313644}

Submitted on 9 Sep 2021

HAL is a multi-disciplinary open access archive for the deposit and dissemination of scientific research documents, whether they are published or not. The documents may come from teaching and research institutions in France or abroad, or from public or private research centers.
L'archive ouverte pluridisciplinaire HAL, est destinée au dépôt et à la diffusion de documents scientifiques de niveau recherche, publiés ou non, émanant des établissements d'enseignement et de recherche français ou étrangers, des laboratoires publics ou privés. 


\title{
Application of the Method of Manufactured Solutions to the verification of a pressure-based finite-volume numerical scheme
}

\author{
João Marcelo Vedovoto, Aristeu da Silveira Neto \\ Faculty of Mechanical Engineering, Federal University of Uberlândia, Uberlândia-MG, Brazil 38400-902 \\ Arnaud Mura \\ Institut Pprime, UPR3346 CNRS - ENSMA - BP40109 - 86961, Poitiers, France \\ Luis Fernando Figueira da Silva \\ Pontifícia Universidade Católica do Rio de Janeiro - Department of Mechanical Engineering - Rio de Janeiro-RJ, \\ Brazil 22453-900
}

\begin{abstract}
The present work provides the description and verification of a pressure-based finite volume approach for variable density flows representative of those encountered in combustion applications. The algorithm under consideration is based on a classical predictor-corrector time integration scheme that employs a projection method for the momentum equations. Spatial discretization is based on a staggered framework with velocity and scalar quantities evaluated in different grids to avoid the rise of checkerboard pressure patterns. The present study then reports a numerical procedure based on a series of tests that make use of the method of manufactured solutions (MMS) and allows to evaluate the effective numerical performance with respect to the theoretical order of accuracy. It is successfully applied to both fully incompressible and variable density flows, confirming again the interest of the method to conduct a preliminary check of the performance of any numerical algorithm.
\end{abstract}

Key words: Variable density flows, Combustion, Low Mach number approximation, Pressure based solver, Code verification, Methods of manufactured solutions

\section{Introduction}

Low Mach number variable density flows arise in several natural as well as technological processes, including meteorological flows or combustion in energy conversion devices (engine, turbojets, etc.). Regarding the prediction and understanding of such a category of flows, the extensive use of mathematical and numerical techniques is unavoidable. Hence, as the mathematical and numerical methods becomes more complex, the procedures of validation and verification of computer codes must evolve as well. The present work aims at demonstrating systematically the procedure of verification of a CFD code designed to perform the numerical simulation of low Mach number flows. Although such a procedure appears as a necessary preliminary step before tackling the computational modeling of turbulent combustion problems of interest to the authors $[1,2,3,4]$, the literature lacks on information over the subject, especially concerning the 
verification of variable density low Mach number flows. In order to clarify the differences between the words verification and validation, we used the following definitions. Verification aims at providing information on whether the mathematical model has been well implemented according to initial design or not, whereas validation refers to how closely the correctly implemented mathematical model mimics a given set of experimental data.

The solutions of the balance equations (mass, momentum, energy, species mass fractions, etc.) are deemed sufficient to represent any flows irrespective of their characteristic velocity, provided that the continuum hypothesis holds, and once suitable constitutive equations for the fluids of interest are provided. However, when dealing with discrete approaches for solving such a system of balance equations, the numerical techniques do involve, invariably, errors. These errors have different sources, spanning from unavoidable roundoff errors to mere programming mistakes (bugs), hence emphasizing the crucial need for well defined procedures to evaluate the numerical accuracy [5]. Moreover, it is of fundamental importance to be able to characterize the capabilities of a numerical approach, i.e., it is imperative to determine whether (or not) the retained mathematical / numerical scheme is suitable to cope with the problem of interest.

Here, we first briefly review the existing strategies that are usually retained to develop compressible and incompressible solvers for low Mach number calculations. Further, we show the mathematical and numerical modeling adopted herein, and then focus on the procedure retained for the verification of low Mach number codes.

\subsection{Compressible vs low Mach number approximations}

An important non-dimensional parameter that characterizes a flow field is the Mach number $M a=U / c$, where $U$ is a characteristic velocity of the flow field, and $c$ is the speed of sound. The incompressible limit is usually defined as the situation associated to Mach number values smaller than 0.3. Above this value the compressibility effects cannot be neglected any longer, and variations of density and pressure are coupled through an equation of state.

For incompressible flows a decoupling between the equations of momentum and the energy equation is possible, and in such flows the pressure variations remain typically very small. The possible density variations are no longer related to pressure variations, but to temperature and variations only. The pressure is often said to be thermodynamically constant, and its influence is only felt through the spatial derivatives that appear in the momentum equation.

In fact, there exists a fundamental difference between the behavior of the set of conservation equations in an incompressible flow, with respect to compressible flows. In compressible flows featuring high Mach number values, the fluid velocity reach the same order of magnitude as the velocity of sound waves, i.e., $O(U) \approx O(c)$, and the mathematical system of governing equations follows an hyperbolic behavior [6], whereas in the low Mach number incompressible case, the velocity of sound waves are much higher than the fluid velocity, i.e., $O(U) \ll O(c)$. Such a disparity of magnitudes between the velocity of the flow and the speed of sound waves leads to a great stiffness in the system of balance equations. This severely hampers the accuracy and convergence of the numerical methods that rely on the consideration of density variations, i.e., density-based solvers [7], which remain the most commonly used to perform the numerical simulations of compressible flows. In the case of incompressible flows such a disparity between the wave and flow velocities is also one of the major sources of numerical instability $[8,9,10]$.

In the present work, we are interested in low velocity flows, i.e., in the incompressible regime, but featuring non negligible density variations, the so-called low Mach number flows. There are 
basically two broad classes of numerical methodologies to deal with this kind of flows: those relying on density-based solvers, i.e., based on methods usually retained for compressible flows, and those relying on pressure-based solvers, such as those retained to perform the numerical simulation of incompressible flows. The application of each one of these two methodologies to low Mach number flows requires the introduction of several modifications and improvements, that are briefly summarized below.

\subsection{Density-based methods}

The density-based methods represent a wide class of numerical schemes originally developed to study compressible flows [11]. Turkel et al. [12] determined that the set of discretized equations retained for the numerical simulation of a compressible flows fails to provide an accurate solution for an incompressible flow. The simulations of incompressible flows based on the fully compressible method, with no modifications to reduce the disparity existing between the flow velocity and the speed of sound, are found impracticable due to the associated computational costs. In this case, temporal integration schemes, whatever they are explicit or implicit, are penalized. In the former case, the Courant-Friedrichs-Lewy (CFL) condition, that must be satisfied at each time step to enforce the numerical stability of the numerical integration scheme, leads to prohibitively small time step values due to the prevailing influence of acoustic waves propagation. In the case of implicit methods such a disparity induces large differences in the characteristic eigenvalues of the algebraic system to be solved, which becomes ill-conditioned, leading therefore to extremely high-cost iterative solutions [13].

Two distinct sets of techniques have been proposed to achieve better convergence properties of density-based solvers, in the limit of low Mach number flows: preconditioning and perturbation methods. Both techniques strive to minimize the stiffness of the algebraic system that results from the discretization of the balance equations.

The first technique pre-multiplies the temporal derivatives by a preconditioning matrix, whose choice is determined according to the problem to be analyzed [7], thus leading to a new set of equations. As a consequence, the initial (stiff) system is altered. The technique essentially aims at re-scaling the characteristic eigenvalues with respect to the original system, so that eigenvalues of similar orders of magnitude can be obtained, leading to a better conditioned system [12, 14]. The major drawback associated with preconditioning methods is that the governing equations are modified in terms of their mathematical nature due to the incorporated transient term. The modified system of equations has only the steady-state solution in common with the original system and becomes therefore devoid of any physical transients. A second limitation is the failure of this methodology, in terms both of efficiency and robustness, in the vicinity of stagnation points, where the characteristic eigenvectors become almost parallel [15]. Furthermore, the design of general purpose pre-conditioners adequate for a large variety of physical problems remains far from being straightforward.

The second set of techniques is the perturbation methods, or asymptotic analysis. In this case, a perturbed form of the equations is used to reduce the stiffness of the algebraic system of equations. A Taylor expansion is performed in terms of the Mach number decoupling the acoustic waves from the equations, and replacing them with a set of pseudo-acoustic forms, where the wave velocities become the same order of magnitude as the fluid velocity. Such a procedure alters the velocity of the acoustic waves in order to allow the numerical integration to be performed 
with larger time steps [7, 13].

Other methodologies have been also developed for the purpose of considering density variations, such as the artificial compressibility methods, and the PGS (Pressure Gradient Scaling). The first, described, for instance in [16], has been successfully applied to the numerical simulation of reactive flows and, in particular, to describe the propagation of a planar turbulent premixed flame [17]. The PGS method [18, 19, 20, 21] displays certain similarities to the perturbation methods, and it also acts on the pressure term that appears in the momentum equation. In this method the pressure gradient is multiplied by a factor $1 / \alpha^{2}$, where $\alpha$ is a constant that amplifies the pressure variations. As a consequence, since the velocity of acoustic waves is decreased by a factor $\alpha$, the pressure variations are amplified by a factor $\alpha^{2}$, thus, improving the robustness of the numerical method as the Mach number tends towards zero [19]. Finally, it is worth adding that one reason for the use of the last two methods rely in the fact that they can be easily implemented within an existing compressible solver.

\subsection{Pressure-based methods}

In contrast to the methodologies discussed above, that are based on density variations, pressurebased methods have been initially proposed to solve fully incompressible flows, retaining the pressure as one of the primary variables. Such numerical schemes that are often referred to as pressure-corrections methods, or projection methods, evaluate the pressure and velocity fields in a segregated manner $[22,23]$. In pressure-based methods, the pressure does not play a thermodynamic role, but ensures the incompressibility condition, which leads to a discretization scheme based on a separation of operators (splitting method). In a first step, momentum equations are solved to obtain an estimated velocity field, based on a previous evaluation of pressure. The velocity field should be solenoidal, and this property is enforced by a subsequent projection step within the subspace of divergence-free vectorial fields. Such projection, which defines the corrector step, relies on the Hodge decomposition theorem [24]. The pioneering works in this field $[22,25]$ have provided the basis for the development of several projection schemes that are still currently used. More specifically, the work of Patankar and co-workers [25], has led to a family of methodologies refereed to as the SIMPLE (Semi-Implicit Pressure Linked Equations) approach, which undoubtedly remains the most widely used to obtain the solution of incompressible flows.

As previously mentioned, in the low Mach number regime, the compressibility effects have a negligible influence on the momentum transport and the pressure is only a weak function of density. To prevent significant inaccuracies when performing the evaluation of pressure, it is usually divided into two distinct components:

$$
P(\mathbf{x}, t)=P_{o}(t)+P^{\prime}(\mathbf{x}, t),
$$

where $P_{o}$ is a reference pressure level ${ }^{1}$, with $P_{o}(t) / P_{o}=O(1)$, and $P(\mathbf{x}, t) / P_{o}=O\left(M a^{2}\right)$. It is worth noting that $P_{o}(t)$ is often referred to as the thermodynamic pressure, whereas $P^{\prime}(\mathbf{x}, t)$ is called the dynamic pressure since it is directly related to modifications of the velocity field.

\footnotetext{
${ }^{1}$ Since we are interested in gaseous flow fields, the effects of gravity are not considered, hence $P_{o}(t)$ is a function of the time only.
} 
Using such a decomposition, the thermodynamic pressure appears in the equations of state and energy conservation only, vanishing in the momentum equation. Since its gradient is zero everywhere, only the gradient of the dynamic pressure component remains. It is worth noting that this procedure significantly accelerates the convergence only if the pressure fluctuations remain sufficiently small.

Since one of the objectives of the present work is to provide guidelines for verification and cross-checks of a recently developed incompressible code, the most natural choice for an extension to variable density flows is a formulation based on pressure variations, i.e., to extend a mathematical and numerical model proposed for fully incompressible flows to handle density variations.

A wide class of numerical methods that remains used to perform such simulations of low Mach number flows, is based on predictor-corrector methods. Several works, [8, 9, 10, 26, 27, 28, 29, 30] share such pressure-velocity coupling. Rider et al. [31] report an extensive discussion about robust projection methods applied to variable density low Mach number flows. The numerical strategy retained for the purpose of the present study is described below.

\section{Mathematical modeling}

Since we intend to simulate variable density flows, we consider a mathematical model suitable for a compressible flow, in which the primary transported variables are the density $\rho(\mathbf{x}, t)$, the three velocity components $u_{i}(\mathbf{x}, t)(i=1,2,3)$, and the temperature. The non-dimensional balance equations for the cited variables in space, $\left(x_{i}, i=1,2,3\right)$, and time, $t$, are summarized below along with an equation of state that relates the thermodynamic component of the pressure to density and temperature. It is worth noting that dimensional quantities are now referred to with the superscript "^’.

$$
\frac{\partial \rho}{\partial t}+\frac{\partial \rho u_{i}}{\partial x_{i}}=S_{\rho}
$$

$$
\frac{\partial \rho u_{i}}{\partial t}+\frac{\partial \rho u_{i} u_{j}}{\partial x_{j}}=-\frac{\partial P}{\partial x_{i}}+\frac{1}{R e} \frac{\partial}{\partial x_{j}} \tau_{i j}+S_{u_{i}},
$$

$$
\tau_{i j}=-\frac{2}{3} \mu \frac{\partial u_{k}}{\partial x_{k}} \delta_{i j}+\mu\left(\frac{\partial u_{i}}{\partial x_{j}}+\frac{\partial u_{j}}{\partial x_{i}}\right),
$$

$$
P=P_{o}(t)+P^{\prime}(\mathbf{x}, t) ; P_{o}=\rho \phi .
$$

The above set of equations is obtained in a non-dimensional form using:

$$
x_{i}=\hat{x}_{i} / L_{r e f}, \quad t=\hat{t} u_{r e f} / L_{r e f}, \quad u_{i}=\hat{u}_{i} / u_{r e f},
$$

$$
P=\frac{\hat{P}}{\left(\rho_{r e f} u_{r e f}^{2}\right)}, \quad \rho=\hat{\rho} / \rho_{r e f},
$$

In Eq. (5), $P_{o}(t)$ is the thermodynamic pressure, a function of time only, $P^{\prime}(\mathbf{x}, t)$ is the dynamic pressure, henceforth denoted only by $p$. In the same equation, the variable $\phi$ stands for a reduced temperature, defined by

$$
\phi=\left(T-T_{u}\right) /\left(T_{b}-T_{u}\right),
$$


where $T_{b}$ (resp. $T_{u}$ ) denotes the maximal (resp. minimal) value of the temperature, in such a manner that $\phi \in[0 ; 1]$. For instance, if we consider a premixed flame propagating towards unburned reactants, $T_{u}$ and $T_{b}$ stand for the temperature in the fresh mixture and fully burned products of combustion respectively. The transport equation for the reduced temperature writes:

$$
\rho C_{p} \frac{\partial \phi}{\partial t}+\rho C_{p} u_{i} \frac{\partial \phi}{\partial x_{i}}=\frac{1}{\operatorname{RePr}} \frac{\partial}{\partial x_{j}}\left(\kappa \frac{\partial \phi}{\partial x_{j}}\right)+\left(\frac{\gamma-1}{\gamma}\right) \frac{d P_{o}}{d t}+S_{\phi},
$$

where $\operatorname{Pr}=\mu_{r e f} C_{p_{r e f}} / \kappa_{r e f}$, and $R e=\rho_{r e f} u_{r e f} L_{r e f} / \mu_{r e f}$, denote the Prandtl and Reynolds numbers.

It is noteworthy that, in the previous set of conservation equations Eqs. (2), (3), and (9), temperature dependent variables, such as the transport properties: viscosity $\mu$, and thermal conductivity $\kappa$, and the heat capacity $C_{p}$, have been made non dimensional with respect to their values at temperature $T_{u}$. The corresponding values are denoted $\mu_{r e f}, \kappa_{r e f}$, and $C_{p_{r e f}}$.

Finally, the source terms $S_{u_{i}}, S_{\rho}$, and $S_{\phi}$ have been included in Eqs. (2), (3), and (9), just for the sake of generality and will be discussed later.

In transient flows the balance equations must be integrated in time as well, which requires the choice of a suitable time marching scheme. Temporal integration schemes are essentially controlled by the Courant criterion $C o=\left(u_{i} \delta t\right) / \delta x_{i}, i=1,2,3$. Explicit schemes exhibit numerical stability issues when using Courant number values larger than unity. However, such a numerical limitation does not apply to implicit or semi-implicit discretizations. Ferziger and Peric [16] provide an introduction to several types of classical temporal integration methods. Examples of semi-implicit approaches can be found in [36], and a detailed evaluation of various semi-implicit schemes has been reported in [37]. The temporal integration schemes retained in the present work are fully implicit, in such a manner that it is possible to reach statistically steady regimes faster than by resorting to explicit time integration techniques.

Arscher et al. [37] proposed a semi-discretized arrangement in such a way that any differential equation could be temporally integrated using second order semi-implicit schemes:

$$
\begin{gathered}
\frac{1}{\Delta t}\left[\left(\gamma+\frac{1}{2}\right) u^{n+1}-2 \gamma u^{n}+\left(\gamma-\frac{1}{2}\right) u^{n-1}\right]=(\gamma+1) \zeta\left(u^{n}\right)-\gamma \zeta\left(u^{n-1}\right)+ \\
{\left[\left(\gamma+\frac{c}{2}\right) \epsilon\left(u^{n+1}\right)+(1-\gamma-c) \epsilon\left(u^{n}\right)+\frac{c}{2} \epsilon\left(u^{n-1}\right)\right],}
\end{gathered}
$$

where $\zeta(u)$ and $\epsilon(u)$ stand for, respectively, diffusive and advective contributions to the differential equation, and $n$ represents the time level. Depending on the values retained for constants $\gamma$ and $c$, different time integration schemes can be obtained [37]:

- Crank-Nicolson Adams-Bashfort (CNAB): $(\gamma, c)=(0.5,0.0)$;

- Modified Crank-Nicolson Adams-Bashfort (MCNAB): $(\gamma, c)=(0.5,0.125)$;

- Crank-Nicolson Leap Frog $(\mathrm{CNLF}):(\gamma, c)=(0.0,1.0)$;

- Semi-Backward Difference Formula (SBDF): $(\gamma, c)=(1.0,0.0)$.

In the present work, instead of a semi-implicit, a fully implicit scheme is adopted. However, the organization allowed by Eq. (10) remains very attractive, since it includes the possibility 
of ( $i$ ) describing several methods of temporal discretization and, hence, (ii) choosing the more adequate for a given problem.

Retaining the same values of the constants $\gamma$ and $c$, Eq. (10) can be adapted within a fully implicit framework as follows:

$$
\begin{array}{r}
\frac{1}{\Delta t}\left[\left(\gamma+\frac{1}{2}\right) u^{n+1}-2 \gamma u^{n}+\left(\gamma-\frac{1}{2}\right) u^{n-1}\right]=\left(\gamma+\frac{c}{2}\right)\left(\zeta\left(u^{n+1}\right)+\epsilon\left(u^{n+1}\right)\right) \\
+(1-\gamma-c)\left(\zeta\left(u^{n}\right)+\epsilon\left(u^{n}\right)\right)+\frac{c}{2}\left(\zeta\left(u^{n-1}\right)+\epsilon\left(u^{n-1}\right)\right),
\end{array}
$$

Applying Eq. (3) in Eq. (11), and re-arranging the different terms, the momentum equation, once discretized in time takes the following form:

$$
\begin{aligned}
& \frac{(\gamma+0.5) \rho^{n+1} u_{i}^{n+1}-(2 \gamma) \rho^{n} u_{i}^{n}+(\gamma-0.5) \rho^{n-1} u_{i}^{n-1}}{\Delta t}=-\frac{\partial p^{n+1}}{\partial x_{i}} \\
&+(\gamma+0.5 c) m_{2}+(1-\gamma-c) m_{1}+(0.5 c) m_{0}+S_{u i}^{n+1},
\end{aligned}
$$

where we defined:

$$
m_{k+1}=\left(\frac{\partial \tau_{i j}^{n+k}}{\partial x_{j}}-\frac{\partial \rho^{n+k} u_{i}^{n+k} u_{j}^{n+k}}{\partial x_{j}}\right), k=-1,0,1
$$

As mentioned previously, the choice of the constant values $\gamma$ and $c$, allows to recover the CrankNicolson method $(\gamma, c)=(0.5,0.0)$, the Modified Crank-Nicolson $(\gamma, c)=(0.5,0.125)$, the Leap Frog method $(\gamma, c)=(0.0,1.0)$ and, the Backward Difference Formula - BDF, $(\gamma, c)=(1.0,0.0)$. Finally, it is wort recalling that, that through Eq. (12), the fully implicity framework retained here requires the numerical resolution of a large algebraic system.

\subsection{Projection method}

Since a fully implicit fractional step method is used in the present work, a Poisson type equation must be solved to apply a pressure correction to both velocity and pressure fields. To provide such an equation, we write Eq. (12), with the pressure derivative evaluated at time level $n$ :

$$
\begin{aligned}
\frac{(\gamma+0.5) \rho^{n+1} \tilde{u}_{i}^{n+1}-}{(2 \gamma) \rho^{n} u_{i}^{n}+(\gamma-0.5) \rho^{n-1} u_{i}^{n-1}} & =-\frac{\partial p^{n}}{\partial x_{i}} \\
& +(\gamma+0.5 c) m_{2}+(1-\gamma-c) m_{1}+(0.5 c) m_{0}+S_{u i}^{n+1},
\end{aligned}
$$

where $\tilde{u}_{i}^{n+1}$ stands for the estimated velocity field. Subtracting Eq. (14) from Eq. (12), defining $Q=p^{n+1}-p^{n}$, and rearranging the different terms, one obtains:

$$
\frac{\rho^{n+1}(\gamma+0.5)}{\Delta t}\left(\tilde{u}_{i}^{n+1}-u_{i}^{n+1}\right)=\frac{\partial Q}{\partial x_{i}},
$$

we then proceed classically by taking the divergence of Eq. (15):

$$
\frac{(\gamma+0.5)}{\Delta t}\left(\frac{\partial \tilde{u}_{i}^{n+1}}{\partial x_{i}}-\frac{\partial u_{i}^{n+1}}{\partial x_{i}}\right)=\frac{\partial}{\partial x_{i}}\left(\frac{1}{\rho^{n+1}} \frac{\partial Q}{\partial x_{i}}\right) .
$$

In situations where the density can be considered to be constant, at least along a streamline, the second derivative at the LHS of Eq. (16) is zero due the incompressibility condition. However, 
when density variations arise from temperature (and potential composition) variations as encountered for instance in reactive flows, such term can no longer be discarded.

In the low Mach number flows under consideration, the density is solely determined by the temperature and thermodynamic pressure fields. The energy equation plays the role of an additional constraint on the velocity field, which is enforced by the dynamic pressure. This constraint acts onto the flow field divergence, and it is related to the total derivative of the density field, which involves - through the equation of state Eq. (5) - the total derivatives of both pressure and temperature. The latter can be expressed thanks to the energy conservation equation, i.e. Eq. (9), thus leading to:

$$
\frac{\partial u_{i}}{\partial x_{i}}+S_{c}=\frac{1}{C_{p} P_{o}}\left\{\frac{1}{\operatorname{RePr}} \frac{\partial}{\partial x_{i}}\left(\kappa \frac{\partial \phi}{\partial x_{i}}\right)+\left(\frac{\gamma-1}{\gamma}-C_{p}\right) \frac{d P_{o}}{d t}+S_{\phi}+C_{p} T S_{\rho}\right\},
$$

where $S_{c}$ is a mathematical source term associated with the constraint itself. This source term, which is added to the constraint, will be used in the subsequent application of the method of manufactured solutions devoted to the low Mach number scheme verification. Its determination will be discussed in the corresponding section. The equation for pressure correction, for low Mach number flows simulations may therefore be written as:

$$
\begin{array}{r}
\frac{\partial}{\partial x_{i}}\left(\frac{1}{\rho^{n+1}} \frac{\partial Q}{\partial x_{i}}\right)=\frac{(\gamma+0.5)}{\Delta t}\left\{\frac{\partial \tilde{u}_{i}^{n+1}}{\partial x_{i}}-\frac{1}{C_{p} P_{o}}\left[\frac{1}{\operatorname{RePr}} \frac{\partial}{\partial x_{i}}\left(\kappa \frac{\partial \phi}{\partial x_{i}}\right)\right]\right\} \\
+\frac{(\gamma+0.5)}{\Delta t}\left[\left(\frac{\gamma-1}{\gamma}-C_{p}\right) \frac{d P_{o}}{d t}+S_{\phi}+C_{p} T S_{\rho}-S_{c}\right]
\end{array}
$$

Once the pressure correction is evaluated, the velocity field can be updated:

$$
u_{i}^{n+1}=\tilde{u}_{i}^{n+1}-\left(\frac{\Delta t}{(\gamma+0.5) \rho^{n+1}}\right) \frac{\partial Q}{\partial x_{i}} .
$$

The constraint given by Eq.(17) in variable density flows has been previously discussed, among others, in [28] and [39]. Moreover, an inner iterative process can be used to enhance both stability and convergence of the solution process. Further comments about such a procedure and its effects on the numerical convergence are deferred to the section devoted to the validation of low Mach number manufactured solutions. Finally, it is worth recalling that, for incompressible flows simulations, the constraint presented above is no longer necessary.

\section{Numerical modeling}

Important factors that directly impact the performances of a numerical method that seeks to solve variable density incompressible flows are: (i) the choice of the variables arrangement in the computational grid, (ii) the type and order of accuracy of the numerical scheme retained to perform the discretization in space of both viscous and advective terms, and (iii) the type and order of accuracy of the method chosen to perform the temporal integration.

If the discretization relies on a finite-volume (FV) approach, it is necessary to evaluate vectorial fields coupled to scalar fields, as it is the case when the Navier-Stokes equations are considered. The issue associated to the positioning of the primary variables in the computational grid 
must be carefully accounted. Several options exist, but they may be classified into two principal groups: staggered or co-located grids. In the former case, the vectorial and scalar variables are positioned in different locations. In the latter, all physical variables are evaluated at the same location. This is the simplest choice for implementation, in particular when distributed computing strategies are to be applied. However, in incompressible flows, if the velocity and pressure are positioned in the same location, decoupling instabilities, often referred to as checkerboard patterns can arise $[6,16,27]$.

The discretization procedure retained for the momentum equation is basically identical for both the staggered and the co-located arrangements. Nevertheless, with the latter, the velocity components, positioned at the center of the control volumes must be interpolated to their faces. The most common procedure is to use the Rhie-Chow interpolation [32]. Shen et al, [33] underline that the Rhie-Chow interpolation produces excellent results when steady-state solutions are sought for, and large time steps used, but pressure field oscillations may arise for small time steps and during transient simulations. Versteeg and Malalasekera [34], and Ferziger and Peric [16], argue that there is no intrinsic physical requirement that all variables share the same location. For cartesian grids, the concept of staggered grid was introduced in reference [35]. If second order of accuracy schemes are retained for spatial discretization, such an arrangement does not require additional interpolations, which otherwise would be necessary for a co-located grid. Finally, the major advantages of the staggered variable arrangement is that the pressure terms are naturally discretized by second order accuracy central differences scheme, without any resort to interpolation rules, and the evaluation of mass fluxes at the faces of control volumes is straightforward, which leads to a strong coupling between pressure and velocity, thus avoiding spurious instabilities in the calculated pressure field.

The numerical method chosen for solving the variable density momentum, reduced temperature and Poisson equations is based on a three-dimensional, conservative, staggered, finitevolume discretization. The central difference scheme (CDS) is applied to express both diffusive and advective contributions of the present finite-volume scheme. Depending on the class of flows simulated, some iterative solvers can fail to converge when applied to the algebraic equation systems derived from central difference approximations of convective fluxes. The reason why it can happen is because grid-to-grid oscillations are not solved by central finitedifference schemes, and hence, should be removed because they can lead to numerical instabilities [40]. Practically, the elimination of these spurious short waves is obtained by introducing artificial dissipation through additional damping terms in the equations [41] or, more efficiently, through filtering [40] without affecting the physical long waves. It is possible yet to apply a deferred correction approach on the advective terms of the balance equations. Such approach designed to improve stability is explained in [16].

A fully implicit approach is adopted, and the resulting linear systems are solved using the MSIP - Modified Strongly Implicit Procedure [42]. The numerical code developed is capable of performing massively parallel distributed computations also. The corresponding parallelization strategy relies on a three-dimensional cartesian topology of domain decomposition, its detailed description is outside the scope of the present study.

The structure adopted for the computational grid, can be non-uniform in such a manner that it is necessary to perform interpolations to discretize any spatial derivatives. Such interpolations are obtained by using classical distance-weighted rules [16] e.g., to determine the value of a scalar property, $\theta$, on a face $e$, the following expression is used: $\theta_{e}=\theta_{E} \Lambda_{e}+\theta_{P}\left(1-\Lambda_{e}\right)$, where: 


$$
\Lambda_{e}=\frac{(\delta x)_{e}^{-}}{(\delta x)_{e}}
$$

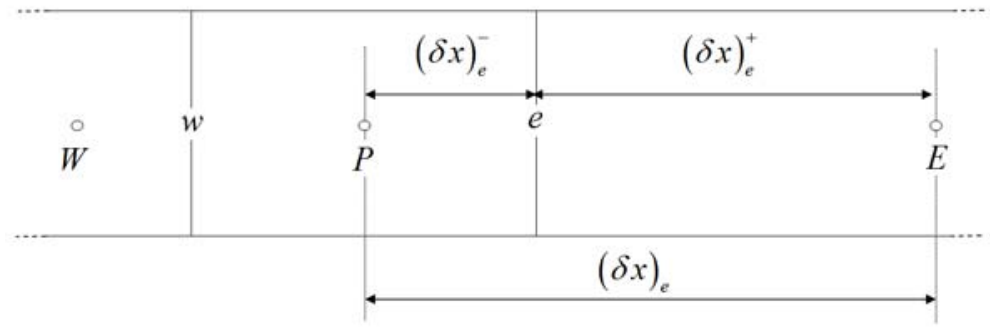

Figure 1: Non-uniform finite-volume grid and distances associated to the face $e$.

The indexes $P, E$ and $e$, are, respectively, the center of the current control volume, the center of the left control volume, and the position of the face of the control volume centered in $P$, that lies between $P$ and $E$, as can be seen in Fig. (1).

The iterative procedure, for each time step, is summarized in Table (1):

Table 1:

Algorithm 1- Projection method with inner iteration procedure

[1] The scalar equation for reduced temperature [Eq. (9)] is advanced in time using using the Crank Nicolson integration scheme, [Eqs. $(12,13)]$ with $(\gamma, c)=(0.5,0.0)$

[2] The equation of state [Eq. (5)] is evaluated, yielding the density in the actual time step

[3] The momentum equations [Eq. (14)] are advanced in time, yielding the estimated velocity field $\tilde{u}_{i}^{n+1}$

[4] The variable-coefficient Poisson equation [Eq. (18)] is solved yielding the pressure correction $Q$, the pressure and velocity fields are updated [Eq.(19)]

[5] The continuity equation [Eq.(2)] is evaluated to check the mass conservation

[6] Returns to item [1], until the cycling process is finished

[7] Time is advanced: $t^{n+1}=t^{n}+\delta t^{n}$

[8] Process is repeated from step [1] until $t^{n+1}=t_{\text {end }}$

It is important to underline that the algorithm described above ensures mass conservation within each single iteration step, however, increasing the number of inner iterations improves both its precision and numerical stability, as will be shown in the following section.

\section{Code verification using the Method of Manufactured Solutions - MMS}

The resort to the method of manufactured solutions is progressively becoming a classical, and well accepted methodology retained in the framework of numerical code verification [43]. There is an undeniable interest in the use of such a method to quantify accurately numerical capabilities before using computational programs to perform the simulation of more complicated physical systems. 
The MMS consists in developing a priori known analytical solutions of the system of governing equations. These manufactured solutions modify the original equations by adding a 'source term', such as those presented in Eqs. (2), (3) and (9). To construct the manufactured solution a set of almost arbitrary functions are selected and substituted into the considered system PDEs, thus allowing to solve its derivatives analytically. The result of such a substitution is the 'source term'. The source term is then considered as an input to the numerical code, so it becomes able to reproduce the manufactured solution. The pioneering works that make use of manufactured solutions with the objective of verifying the order of accuracy of a given numerical code can be assigned to Steinberg and Roache [43], Roache et. al. [44] and Roache [45].

In order to obtain the order of accuracy of the developed numerical implementation, a quantitative metric of the error is chosen, and successive grid refinements are performed. As the error metric decays with the grid refinement, it is evaluated as a function of the characteristic mesh size $h$.

The arbitrary nature of the analytical functions must satisfy, at least, the following conditions:

- They must be continuous smooth function of independent variables (problems involving discontinuities are generally dealt with using lower order algorithms due to stability issues);

- The solution must be continuously differentiable up to the order required by the corresponding terms in the governing equations;

- To avoid numerical difficulties, manufactured solutions should avoid negative values for quantities that are physically defined as positive semi-definite (e.g. density, molecular viscosity);

- If periodic boundary conditions are chosen, the solution must be periodic as well to avoid the development of discontinuities.

Salari and Knupp [46] provide a well documented guideline for creating manufactured solutions as well as the procedure for obtaining the corresponding source terms and further analysis of the results.

For a given level of resolution, we define $\Phi_{(i, j, k)}^{h}$ as the discrete value of any variable $\Phi$ of interest, e.g. the density, velocity components, pressure or reduced temperature, at any point $(i, j, k)$, and $\Phi_{(i, j, k)}^{e}$ the corresponding value of the manufactured solution. The first step of the verification procedure now requires to define a metric of the numerical error, denoted $\Psi_{h}$ in the following, in order to quantify subsequently the error decay rate obtained for decreasing values of the characteristic grid mesh size $h$.

Retaining the $L_{2}$-norm as a relevant metric of the numerical error, we introduce:

$$
\Psi_{h}=L_{2}\left(\Phi_{(i, j, k)}^{h}\right)=\sqrt{\frac{1}{N} \sum_{i, j, k}\left(\Phi_{(i, j, k)}^{h}-\Phi_{(i, j, k)}^{e}\right)^{2}},
$$

where $N$ denotes the total number of grid points.

The ratio of error decay is defined according to:

$$
r_{e}=\log \left(\frac{\Psi_{2 h}}{\Psi_{h}}\right),
$$


with $\Psi_{2 h}$ the numerical value of the metric error obtained on a grid with a characteristic mesh size $2 h$.

Following the nomenclature retained in [46], we define also the order of accuracy $q$ as:

$$
q \approx \frac{r_{e}}{\log (2)}
$$

Using Eq. (23), it is possible to verify that the characteristic error decay ratio, using grids with characteristics mesh sizes $2 h$ and $h$, for methods of order of accuracy $q=1,2$ and 3 , must be, approximately, 2, 4 and 8, respectively. It is worth noting that, although possible, the value of $r_{e}$ does not always converge monotonically as the mesh is progressively refined, and it may exhibit some oscillations.

The process of verification presented herein is divided into two distinct parts. First, the numerical code is tested using a manufactured solution that aims at verifying the order of accuracy for an incompressible solution (zero Mach number limit). This is performed using solutions based on sine and cosine functions, built in such a manner that the velocity divergence is zero. A second set of tests aims at mimicking the propagation of a corrugated flame front that separates heavy from light gases. The application of the method of manufactured solutions still remains more scarce for such low Mach number situations. If we except the expression of the equation of state, the solution retained to perform this analysis is the same as the one previously considered in the recent studies conducted by Shunn and Ham [47], and Shunn and Knupp [48]. This second set of tests clearly aims at evaluating the numerical capabilities of the developed code to deal with variable density flows as those encountered in combustion problems of interest to the authors.

\subsection{Verification of an incompressible solution}

Following the recent work conducted by [49] and [50], the set of functions reported below is retained as a possible solution for velocity, pressure and scalar fields in the limit of a zero Mach number scheme:

$$
\begin{gathered}
u_{e}=\sin ^{2}(2 \pi x+2 \pi y+2 \pi z+t), \\
v_{e}=\cos ^{2}(2 \pi x+2 \pi y+2 \pi z+t), \\
w_{e}=1, \\
p_{e}=\cos (2 \pi x+2 \pi y+2 \pi z+t), \\
\phi_{e}=K_{\phi_{1}}+\frac{1}{K_{\phi_{2}}}+\cos (2 \pi x+2 \pi y+2 \pi z+t) .
\end{gathered}
$$

In the previous equations $K_{\phi_{1}}$ and $K_{\phi_{2}}$ are constant parameters, $t$ is the time. The subscript $e$ stands for the manufactured solutions of the primary variables, i.e. the three velocity components, pressure and reduced temperature

The computational domain retained for the present numerical simulations is a cube of dimensions $[0,1] \times[0,1] \times[0,1]$, in $x, y$ and $z$ directions respectively. The time step is set constant and equal to $10^{-4}$. The parameters $K_{\phi_{1}}$ and $K_{\phi_{2}}$ are fixed respectively to 1 and 0.5 . The constant values of density and viscosity are set to unity. The variable coefficient Poisson solver is used, however, no constraint is enforced for such incompressible solution. Table (2) reports the grid refinement, the decay of $L_{2}$ norm and the resulting order of accuracy $q$. 
Table 2:

Order of accuracy for Dirichlet boundary conditions and constant physical properties.

\begin{tabular}{ccccccccc}
\hline Domain & $16^{3}$ & $q$ & $32^{3}$ & $q$ & $64^{3}$ & $q$ & $128^{3}$ & $q$ \\
\hline$L_{2} u$ & $1.60 \mathrm{E}-02$ & - & $3.80 \mathrm{E}-03$ & 2.07 & $9.30 \mathrm{E}-04$ & 2.03 & $2.33 \mathrm{E}-04$ & 2.00 \\
$L_{2} v$ & $1.60 \mathrm{E}-02$ & - & $3.80 \mathrm{E}-03$ & 2.07 & $9.30 \mathrm{E}-04$ & 2.03 & $2.33 \mathrm{E}-04$ & 2.00 \\
$L_{2} w$ & $3.42 \mathrm{E}-03$ & - & $7.98 \mathrm{E}-04$ & 2.10 & $1.94 \mathrm{E}-04$ & 2.04 & $4.86 \mathrm{E}-05$ & 2.00 \\
$L_{2} p$ & $1.82 \mathrm{E}-01$ & - & $5.44 \mathrm{E}-02$ & 1.74 & $1.52 \mathrm{E}-02$ & 1.84 & $4.10 \mathrm{E}-03$ & 1.89 \\
$L_{2} \phi$ & $1.54 \mathrm{E}-02$ & - & $4.10 \mathrm{E}-03$ & 2.08 & $8.88 \mathrm{E}-04$ & 2.03 & $2.29 \mathrm{E}-04$ & 1.96 \\
\hline
\end{tabular}

The results gathered in Table (2) are illustrated in Figure (2). For the incompressible solution, the numerical code is shown to yield at least second order of accuracy for velocity. For the scalar variable $\phi$, second order of accuracy is achieved as well, although minor oscillations arise. Finally, concerning the pressure, it is possible to note a continuous increase of the order of accuracy as the computational mesh is refined, however, its value does not exceed 1.89.

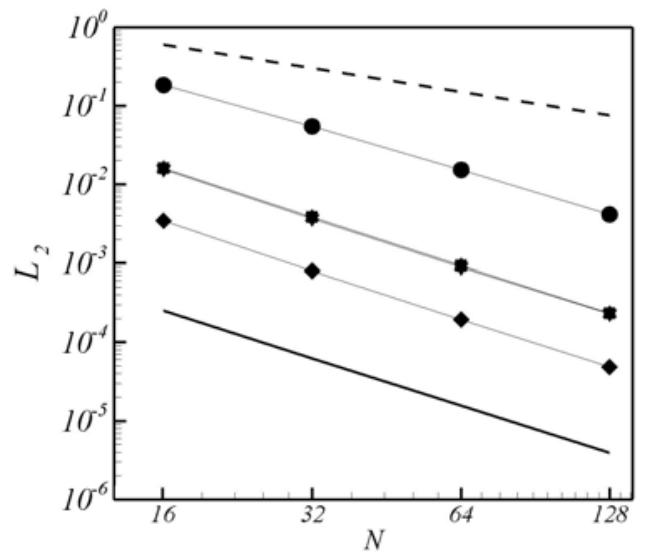

Figure 2: $L_{2}$ norm of the Zero Mach Number manufactured solution. $\mathbf{\square}:$ u, $\mathbf{\Delta}: \mathrm{v}, \boldsymbol{\bullet}: \mathrm{w}, \mathbf{\nabla}: \phi, \bullet$ : p. The solid line stands for the second order decay, and the dashed line stands for first order decay

Such an analysis of the incompressible solution under consideration clearly demonstrates that, in terms of numerical accuracy, the numerical code we developed is a good candidate to perform Large Eddy Simulations of constant density turbulent flows that requires at least second order accuracy.

The preliminary key step associated with the verification of the numerical code being performed for an incompressible regime, we will now turn to variable density flows.

\subsection{Verification of the Low-Mach number solution}

The set of manufactured solutions retained in the present section are similar to those introduced in the previous studies by Shunn and Knupp [48] and Shunn and Ham [47]. However, it is used here with a different equation of state, which involves a different pressure-velocity coupling. It is also worth mentioning that we are interested in density ratio variations representative 
of those encountered in flames, which are significantly smaller than those reported in [47].

The manufactured solutions are not necessarily related to an expected physical solution, however, a solution that attempts to represent some relevant features of a given problem becomes not only a tool to assess the order of accuracy, but also provides a pertaining preview of the behavior of the numerical code, when applied to situations of interest. In this sense, the set of analytical functions reported below gathers some interesting features of a propagative combustion front.

$$
\begin{gathered}
\phi_{e}=\frac{1+\tanh [b \hat{x} \exp (-\omega t)]}{\left(1+\frac{\rho_{0}}{\rho_{1}}\right)+\left(1-\frac{\rho_{0}}{\rho_{1}}\right) \tanh [b \hat{x} \exp (-\omega t)]}, \\
\rho_{e}=\frac{P_{o}}{\phi_{e}} \\
u_{e}=\frac{\rho_{1}-\rho_{0}}{\rho_{e}}\left\{-\omega \hat{x}+\frac{\omega \hat{x}-u_{f}}{\exp [2 b \hat{x} \exp (-\omega t)]+1}+\frac{\omega \log [\exp (2 b \hat{x} \exp (-\omega t))+1]}{2 b \exp (-\omega t)}\right\},
\end{gathered}
$$$$
v_{e}=v_{f} \text {, }
$$$$
w_{e}=0 \text {, }
$$$$
p_{e}=0
$$

where, $\hat{x}=u_{f} t-x+a \cos \left[k_{2}\left(v_{f} t-y\right)\right]$ and $a, b, k_{2}, \omega, u_{f}, v_{f}$ and $P_{o}$ are constant parameters.

It is important to point out that the manufactured solution must be compatible with the full set of equations that governs the evolution of the system under consideration, and this also includes the equation of state and the resulting constraint that applies to the velocity field through Eq. (17). The above manufactured solution does not fulfill such a requirement, and the source term $S_{c}$, previously introduced in Eqs. (17) and (18), must be now considered. Its expression, which is a rather cumbersome combination of exponential and hyperbolic functions, is not reported here. It is evaluated in the same manner as $S_{u_{i}}$ and $S_{\phi}$, i.e. the exact functions (Eqs. (29-34)) are substituted in Eq. (17), and, after solving the derivatives analytically, one obtains the expression of $S_{c}$. Equations (29-34) satisfy the mass balance equation with $S_{\rho}=0$, however, non-zero source terms also appear in momentum, $\left(S_{u_{i}}\right)$, and reduced temperature transport equation $\left(S_{\phi}\right)$.

Table 3:

Values of the constant parameters for the variable density numerical simulation.

\begin{tabular}{cc|cc}
\hline parameter & value & parameter & value \\
\hline$\rho_{o}$ & 5 & $a$ & $1 / 5$ \\
$\rho_{1}$ & 1 & $b$ & 20 \\
$u_{f}$ & 0.25 & $k_{2}$ & $4 \pi$ \\
$v_{f}$ & 0 & $\omega$ & 1 \\
$w_{f}$ & 0 & $\kappa=\mu$ & $10^{-4}$ \\
$P_{o}$ & 5 & $C_{p}$ & 1 \\
\hline
\end{tabular}

Figure (3) displays the temporal evolution of the density field. It is possible to note that, as the time passes, the front is simultaneously advected and diffused. The computational domain is 
a box of $[-1,2] \times[-0.5,0.5] \times[-h / 2, h / 2]$. Five different grids are used, from: $150 \times 50 \times 1$, up to, $2400 \times 800 \times 1$. These grids are henceforth denoted, respectively, by: $4 h, 2 h, h, h / 2$ and $h / 4$. For velocity components, reduced temperature and density, Dirichlet boundary conditions are set at $x=0, y=-1 / 2$ and $y=1 / 2$. A Neumann boundary condition is used in the outlet $x=2.0$. For the pressure, Neumann boundary conditions are applied at $x=0, y=-1 / 2$ and $y=1 / 2$. At the outlet a Dirichlet condition is retained. For all variables, periodicity is assumed in spanwise, (z), direction. Table 3 presents the values considered in this work for simulation of Eqs. (29-31).

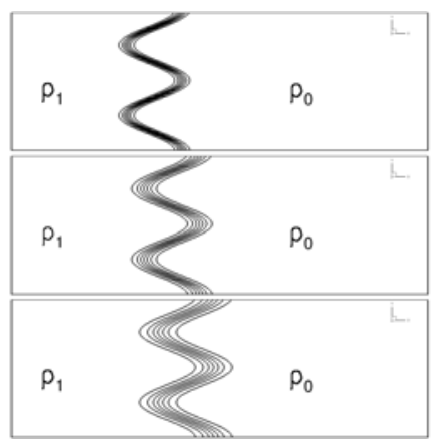

Figure 3: Evolution of the density field. Top to bottom, $t=0.0, t=0.5$ and $t=1.0$. From left to right, each isolines stands for an increase of 0.5 in density from $\rho_{1}=1$, until $\rho_{0}=5$.

The procedure of cycling some parts of the algorithm is used to enhance convergence and stability of the numerical scheme. Such procedure is based on the recent work of Shunn and Ham [47], but with some modifications. First a different equation of state is used, second a constraint on the divergence of the velocity field is added herein, as a part of the strategy retained to solve the Poisson problem. Finally, it is worth recalling that we are interested in density ratio values smaller than those considered in [47].

\subsubsection{Unsteady error behavior}

In this section we discuss the effects of the outer iterative procedure i.e. number of times that the balance, state and the Poisson equations are solved, before the numerical time integration scheme. The influence of the Courant number value on the evolution of the $L_{2}$ norm is also assessed.

In order to quantify only the effects of spatial errors and cycling procedure in the analysis of order of accuracy of low Mach number MMS problems, a Courant number value is chosen, and then, the grid refinement procedure is performed and the subsequent analysis of order of accuracy is conducted. 

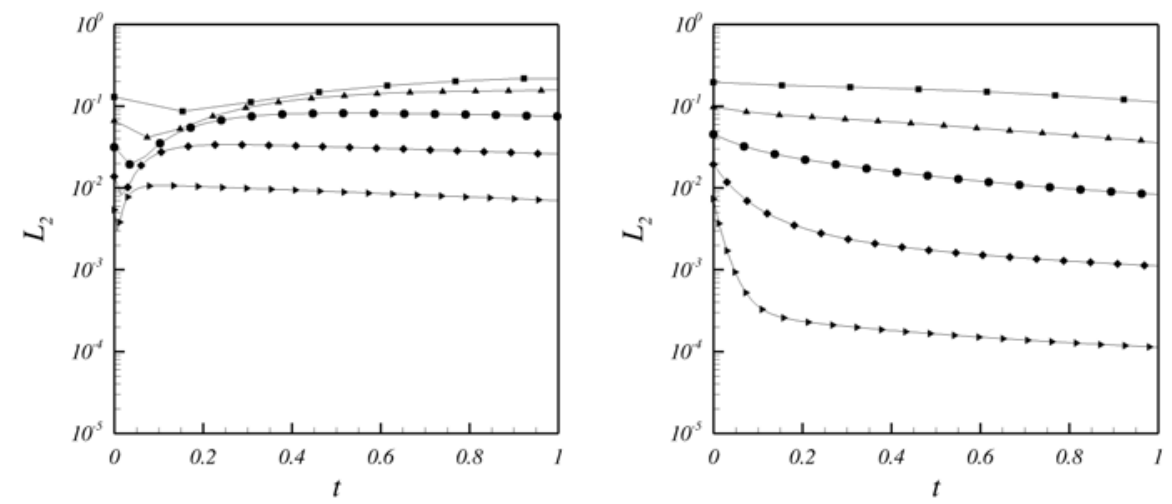

Figure 4: Evolution in time of the $L_{2}$ norm for the density variable with 1 (left) and 10 (right) cycles

$\checkmark: h / 2, \downarrow: h / 4$. For the sake of better visualization, the $L_{2}$ axis is in log scale

Figure (4) shows the unsteady evolution of the $L_{2}$ norm evaluated from the density field. In this case, the Courant number value for the simulations is set to 2 . It is noteworthy that, specially for the refined grids, the increase in the number of cycles leads to a slight decrease of the magnitude of the $L_{2}$ norm and therefore an improvement in order of accuracy. It is also worth noting that such a behavior is also observed for the variables $u, v$ and $\phi$. In figure (5) the evolution of the $L_{2}$ norm of pressure is reported. Unlike the other primary variables of the system, as the number of cycles increases, the magnitude of the $L_{2}$ norm increases as well. However, it will be shown in the next section devoted to the analysis of order of accuracy for the low Mach number MMS that, even with such a negative sensitivity to the number of cycles, its order of accuracy is not penalized.

Although not shown here for the sake of conciseness, we have also found that the $L_{2}$ norm of primary variables errors smoothly decays with time as the front diffuses and the number of control volumes within the front increases. In fact, this is the reason that explains why we stopped the numerical simulations at $t=1.0$. 

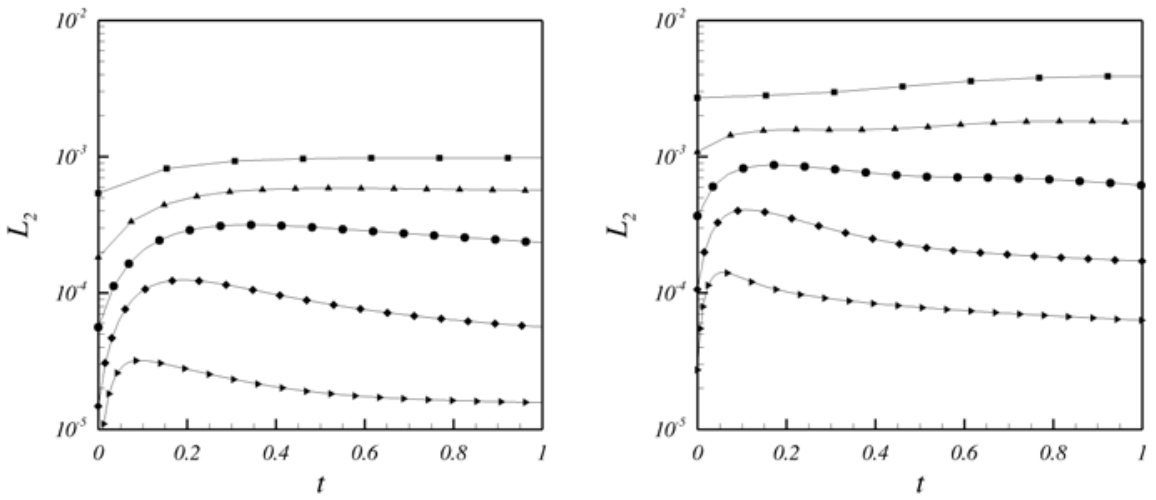

Figure 5: Evolution in time of the $L_{2}$ norm for $p$ with 1 (left) and 10 (right) cycles. For the sake of better visualization, the $L_{2}$ axis is displayed in log scale

Figure (6) displays $L_{2}$ norm for different number of cycles for the $h$ grid. The results clearly show that with higher values of Courant number, the use of more than 5 iterations in the cycling procedure does not provide any further gain in the reduction of error magnitude. However, for Courant number values up to unity, an increase in the number of cycles still gives rise to an improvement of the results.
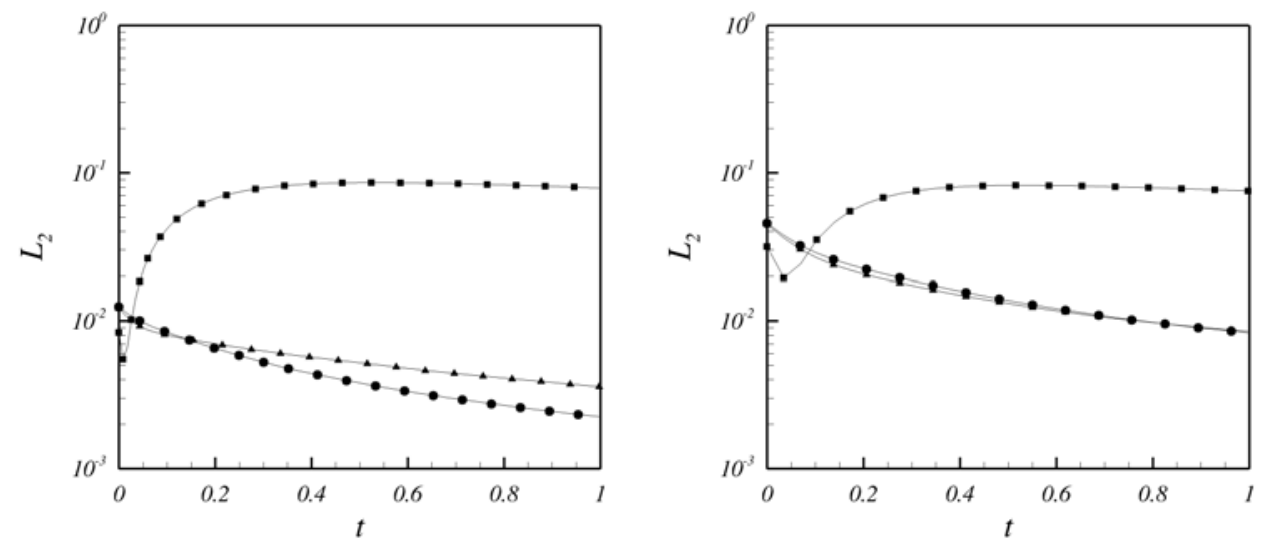

Figure 6: Decay of the $L_{2}$ norm according to the number of cycles $C F L=0.5$ (left) and $C F L=2.0$ (right) for density. 口: Single cycle, $\mathbf{\Delta}: 5$ cycles, $\bullet: 10$ cycles. For the sake of better visualization, the $L_{2}$ axis is plotted in $\log$ scale

\subsubsection{Order of accuracy analysis}

In this section we present and discuss the results of an order of accuracy analysis performed for the low Mach number MMS case. All the simulations presented below have been carried out with a constant value of the Courant number. Although the errors quantified herein by means of 
the $L_{2}$ norm is a rather complicated combination of both spatial and temporal approximations, performing the grid refinement with such a constant Courant number value allows to focus on the influence of the spatial discretization only, and this despite the presence of a residual temporal error since it will remains the same, provided that the Courant number value is kept constant.

Table 4:

Order of accuracy for low Mach number solution, Single cycle, $\mathrm{Co}=2$.

\begin{tabular}{ccccccccccc}
\hline Domain & $4 h$ & $q$ & $2 h$ & $q$ & $h$ & $q$ & $h / 2$ & $q$ & $h / 4$ & $q$ \\
\hline$L_{2} u$ & $8.82 \mathrm{E}-03$ & - & $4.52 \mathrm{E}-03$ & 0.96 & $1.65 \mathrm{E}-03$ & 1.45 & $2.29 \mathrm{E}-04$ & 2.85 & $1.91 \mathrm{E}-05$ & 3.58 \\
$L_{2} v$ & $3.06 \mathrm{E}-03$ & - & $1.28 \mathrm{E}-03$ & 1.25 & $3.27 \mathrm{E}-04$ & 1.97 & $3.47 \mathrm{E}-05$ & 3.24 & $3.44 \mathrm{E}-06$ & 3.33 \\
$L_{2} p$ & $9.82 \mathrm{E}-04$ & - & $5.69 \mathrm{E}-04$ & 0.79 & $2.44 \mathrm{E}-04$ & 1.22 & $5.89 \mathrm{E}-05$ & 2.05 & $1.59 \mathrm{E}-05$ & 1.89 \\
$L_{2} \phi$ & $1.03 \mathrm{E}-01$ & - & $6.73 \mathrm{E}-02$ & 0.61 & $2.39 \mathrm{E}-02$ & 1.50 & $7.70 \mathrm{E}-02$ & 1.01 & $1.40 \mathrm{E}-03$ & 2.07 \\
$L_{2} \rho$ & $2.18 \mathrm{E}-01$ & - & $1.55 \mathrm{E}-01$ & 0.49 & $7.70 \mathrm{E}-02$ & 1.01 & $2.69 \mathrm{E}-02$ & 1.52 & $7.31 \mathrm{E}-03$ & 1.88 \\
\hline
\end{tabular}

Tables (4), (5) and (6) display the order of accuracy, and the value of the $L_{2}$ norm obtained for velocity, density and reduced temperature for different numbers of cycles and a Courant number value $\mathrm{Co}=2$. These values of error and order of accuracy are measured at $t=1.0$ [47].

Table 5:

Order of accuracy for low Mach number solution, 5 cycles, $\mathrm{Co}=2$.

\begin{tabular}{ccccccccccc}
\hline Domain & $4 h$ & $q$ & $2 h$ & $q$ & $h$ & $q$ & $h / 2$ & $q$ & $h / 4$ & $q$ \\
\hline$L_{2} u$ & $8.19 \mathrm{E}-03$ & - & $2.50 \mathrm{E}-03$ & 1.71 & $5.01 \mathrm{E}-04$ & 2.32 & $7.55 \mathrm{E}-05$ & 2.73 & $1.00 \mathrm{E}-05$ & 2.92 \\
$L_{2} v$ & $3.52 \mathrm{E}-03$ & - & $1.17 \mathrm{E}-03$ & 1.60 & $2.29 \mathrm{E}-04$ & 2.35 & $2.82 \mathrm{E}-05$ & 3.02 & $5.85 \mathrm{E}-06$ & 2.27 \\
$L_{2} p$ & $2.68 \mathrm{E}-03$ & - & $1.24 \mathrm{E}-03$ & 1.11 & $4.49 \mathrm{E}-04$ & 1.47 & $1.36 \mathrm{E}-04$ & 1.72 & $6.07 \mathrm{E}-05$ & 1.17 \\
$L_{2} \phi$ & $5.05 \mathrm{E}-02$ & - & $1.16 \mathrm{E}-02$ & 2.12 & $1.96 \mathrm{E}-03$ & 2.57 & $2.98 \mathrm{E}-04$ & 2.72 & $4.87 \mathrm{E}-05$ & 2.61 \\
$L_{2} \rho$ & $1.15 \mathrm{E}-01$ & - & $3.83 \mathrm{E}-02$ & 1.59 & $8.97 \mathrm{E}-03$ & 2.09 & $1.32 \mathrm{E}-03$ & 2.76 & $1.76 \mathrm{E}-04$ & 2.91 \\
\hline
\end{tabular}

Unlike the order of accuracy analysis conducted for the incompressible solution, where the values of the $L_{2}$ norm decrease with a constant order of accuracy, the present results show that, as the grid is refined, not only the magnitude of the $L_{2}$ norm decreases, but also the order of accuracy increases. Such a trend is even more pronounced for velocity, density and reduced temperature when increasing the number of cycles. On the contrary, as far as the pressure field is concerned, the magnitude of the $L_{2}$ norm also increases for an increasing number of cycles.

Table 6:

Order of accuracy for low Mach number solution, 10 cycles, $\mathrm{Co}=2$.

\begin{tabular}{ccccccccccc}
\hline Domain & $4 h$ & $q$ & $2 h$ & $q$ & $h$ & $q$ & $h / 2$ & $q$ & $h / 4$ & $q$ \\
\hline$L_{2} u$ & $8.50 \mathrm{E}-03$ & - & $2.58 \mathrm{E}-03$ & 1.72 & $4.52 \mathrm{E}-04$ & 2.51 & $6.59 \mathrm{E}-05$ & 2.78 & $9.73 \mathrm{E}-06$ & 2.82 \\
$L_{2} v$ & $3.14 \mathrm{E}-03$ & - & $1.02 \mathrm{E}-03$ & 1.63 & $2.05 \mathrm{E}-04$ & 2.31 & $2.12 \mathrm{E}-05$ & 3.27 & $4.24 \mathrm{E}-06$ & 2.32 \\
$L_{2} p$ & $3.90 \mathrm{E}-03$ & - & $1.81 \mathrm{E}-03$ & 1.11 & $6.44 \mathrm{E}-04$ & 1.49 & $1.76 \mathrm{E}-04$ & 1.87 & $6.49 \mathrm{E}-05$ & 1.44 \\
$L_{2} \phi$ & $4.19 \mathrm{E}-02$ & - & $8.34 \mathrm{E}-03$ & 2.33 & $1.26 \mathrm{E}-03$ & 2.73 & $1.32 \mathrm{E}-04$ & 3.25 & $1.29 \mathrm{E}-05$ & 3.35 \\
$L_{2} \rho$ & $1.21 \mathrm{E}-01$ & - & $3.97 \mathrm{E}-02$ & 1.61 & $5.55 \mathrm{E}-03$ & 2.84 & $1.17 \mathrm{E}-03$ & 2.24 & $1.19 \mathrm{E}-04$ & 3.31 \\
\hline
\end{tabular}

Figure (7) displays the order of accuracy achieved for both the pressure and the $u$-component of the velocity. The simulations are carried out using a constant value of the Courant number value $\mathrm{Co}=2$. From a careful examination of Tables (4), (5) and (6), and Figure (7), one can notice that the error decay is larger in single cycle simulations. A similar analysis (not reported) 


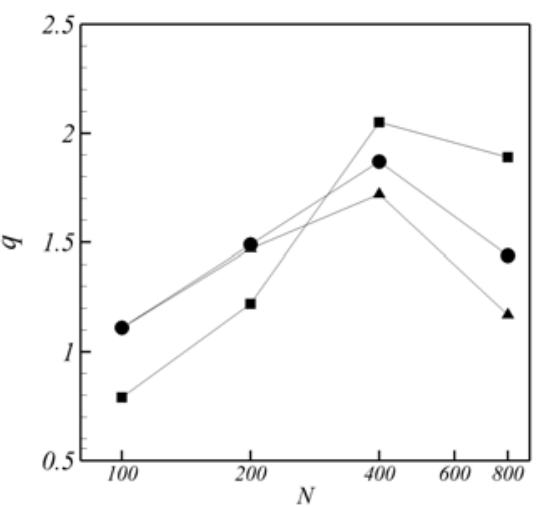
the primary variables.

demonstrates that such a conclusion also holds for different Courant number values, and for all

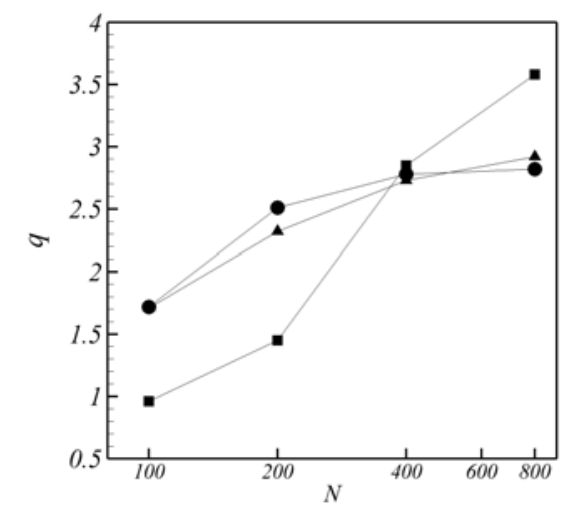

Figure 7: Evolution of the order of accuracy $q$. Pressure (left), $u$ (right). $\mathbf{\square}$ : Single cycle, $\mathbf{\Delta}: 5$ cycles, $\bullet$ : 10 cycles. For the sake of better visualization, the $N$ axis is plotted in log scale

The decays of the $L_{2}$ norm obtained for the primary variables, corresponding to the results gathered in Tables (4) - (6), are illustrated in Figure (7). In this figure, one can note a higher value of the order of accuracy as the number of cycles is increased. Even for the pressure, that is less sensitive to the number of cycles than the others variables, an increase in the order of accuracy is observed.

With regard to the effects of the number of cycles with respect to the order of accuracy, it is worth noticing that, as the grid mesh size is halved from $h$ to $h / 2$, and with a Courant number value $\mathrm{Co}=2$, the order of accuracy for the density is found to increase from 1.52 (Tab. 4, column 8 , line 5) to 2.24 (Tab. 6 , column 8 , line 5), provided that the number of cycles is increased from 1 to 10 .
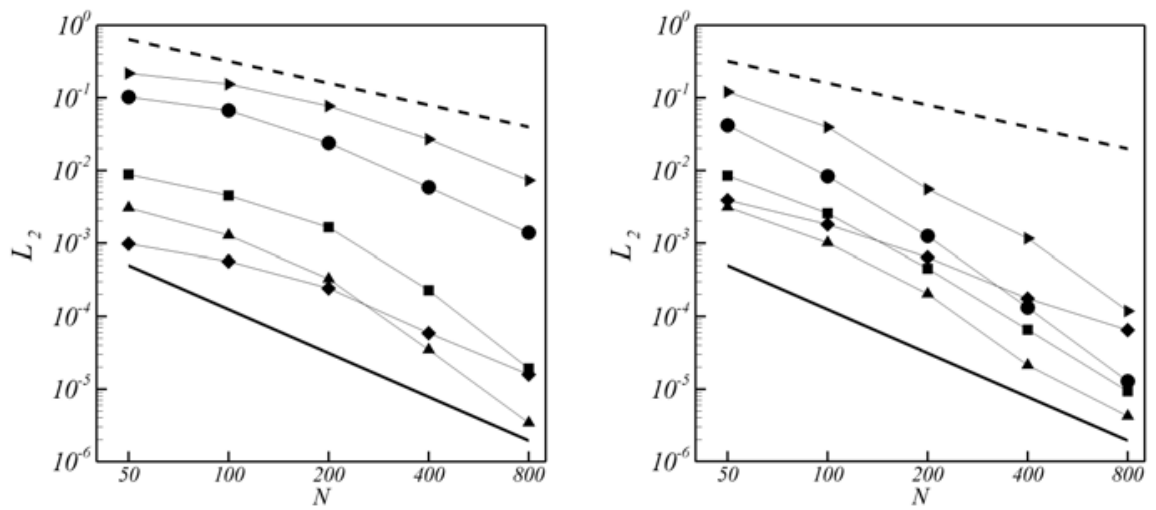

Figure 8: Evolution in time of the $L_{2}$ norm. Single cycle (left), 10 cycles (right). $\mathbf{\square}: \mathrm{u}, \mathbf{\Lambda}: \mathrm{v}, \boldsymbol{\vee}: \mathrm{p}, \bullet: \phi, \boldsymbol{\bullet}$. The solid line stands for the second order decay, and the dashed line stands for first order decay. 

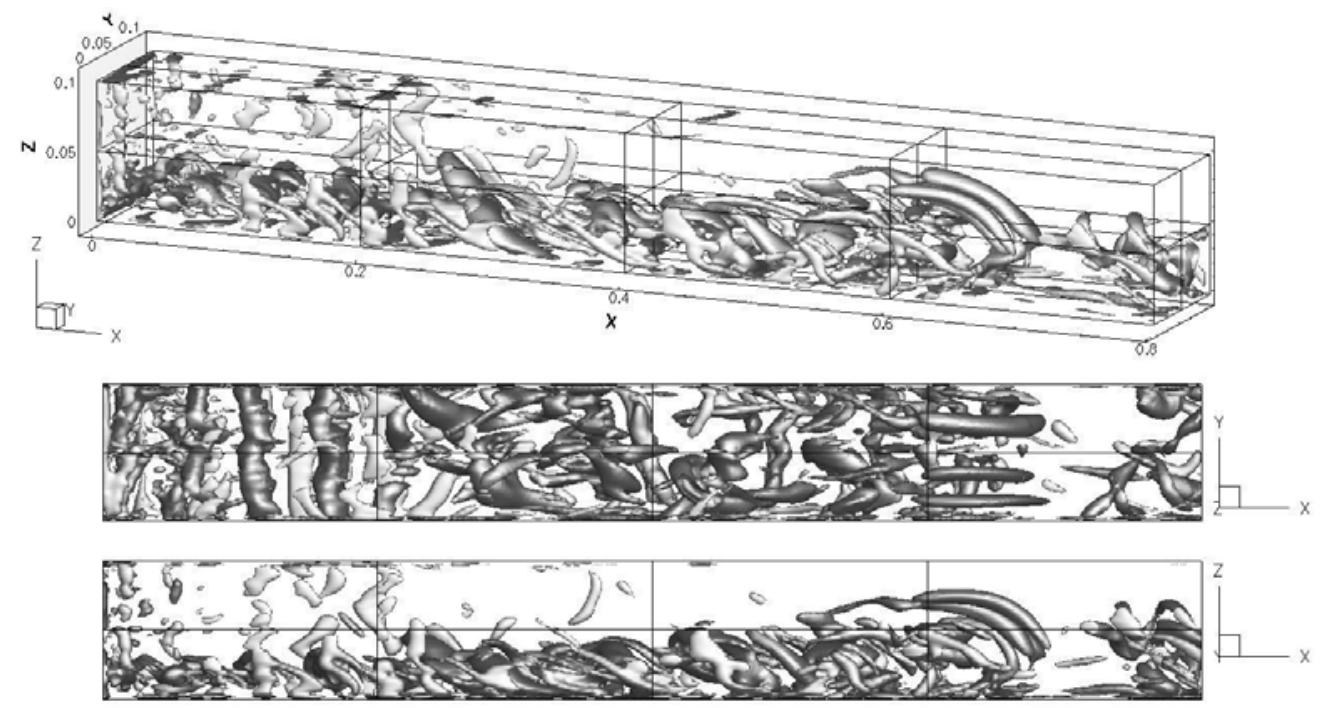

Figure 9: Iso surfaces of $\mathrm{Q}=2^{6}$, at $t=0.46 s$ (46000 iterations). The Reynolds number is 94540

where, $\bar{u}_{q}$ and $\bar{u}_{p}$ are respectively the mean velocities of the auxiliary burner and of the main duct; $h_{p}$ is the height of auxiliary duct. $\delta_{m}$ is the width of the mixing layer. The main duct incoming flow is characterized by a maximum longitudinal velocity of $65 \mathrm{~m} / \mathrm{s}$. The auxiliary duct incoming flow presents a maximum longitudinal velocity of $130 \mathrm{~m} / \mathrm{s}$. The Reynolds number, based on the main duct incoming flow, on its height $\left(h_{q}=80 \mathrm{~mm}\right)$ and on the value of the kinematic viscosity of air at $600 \mathrm{~K}$ is 94540 . 
dynamics is the correct and realistic impositions of boundary conditions, especially inflow and outflow conditions. For the example here described the we retain a methodology for generation realistic inlet boundary conditions based of digital filters. Such an approach involves to create a entire set of random number data which can then be processed using digital filters in such a way that the data presents desired statistical properties such as spatial and temporal correlations. An interesting feature of this approach is the ability of generating anisotropic turbulence, which is fundamental in Large Eddy Simulations [54].

A instantaneous snapshot of the three dimensional simulation of the flow just cited is shown in Figure (9). The snapshot was taken at $t=0.46 s$, equivalent to 46000 iterations. An interesting way to evidencing rotational structures is through the property Q [55]. In Figure (9) it is possible to see the isosurface of the property $Q=2^{6}$. The high value of such property in this case is due the fact that the flow is a high velocity, high Reynolds number, confined flow. The Kelvin-Helmholtz structures, characteristic of mixing layers are notable near the inlet of the domain. The strong three-dimensionality of the flow is also present, in a major part due the turbulence inlet generator.

The fact that we firstly had a totaly verified numerical code was fundamental in the simulation of the above mixing layer. It shows that even tough the MMS are normally applied with smooth well known problems, it does serves (and should be used) as a primary test before coping with problems physically more demanding.

\section{Conclusion}

We have described a procedure for code verification (i) to determine whether, or not, a numerical code solves its governing equations correctly, and (ii) to preview how such a numerical scheme behaves when dealing with real problems. The numerical procedure relies on the manufacture of a general solution of the system of governing equations. Generality of the solution is essential because it guarantees that few, if any, code capabilities will remain unverified.

Two MMS problems are considered. First, a fully incompressible manufactured solution is proposed, achieving the expected order of accuracy. Second, an analytical propagating front separating heavy from light gases is considered. A variable coefficient Poisson solver is used to simulate the corresponding low Mach number flow. A physically consistent constraint is used to ensure that the velocity field is solved correctly. Such a combination of the velocity constraint and the variable-coefficient Poisson solver is found of fundamental importance to ensure both the numerical stability and the expected order of accuracy. An inner iteration procedure is implemented, and despite its high numerical cost, the improvements in terms of both the order of accuracy and error magnitude are undeniable. In this respect, further investigations may be carried out since although possible, the order of accuracy $q$ does not always converge monotonically.

The present results also suggest that determining the optimal operating conditions in terms of grid size, Courant number value, number of outer iterations, etc. is a nontrivial and problemdependent task that deserves more attention than currently afforded.

Finally, although not fully physically consistent - since the MMS is more a mathematical exercise rather than a real attempt of reproducing a real life experiment or physical problem - the procedure of verification allows to gain more insights into the capabilities and limitations of the numerical code we presented to deal with variable density flow fields representative of those encountered in situations relevant of combustion. 


\section{ACKNOWLEDGEMENTS}

This work was performed while L.F. Figueira da Silva was on leave from Centre National de la Recherche Scientifique, Poitiers (France). The authors would like to thank CNPq and PETROBRAS S.A. for the financial support.

\section{References}

[1] Andrade FO, Figueira da Silva LF, Mura A. A hybrid LES/SGS-FDF computational model for turbulent premixed combustion. Proceedings of 20th International Congress of Mechanical Engineering, Gramado, RS, Brazil. November 15-20, 2009.

[2] Mura A, Demoulin FX. Lagrangian intermittent modeling of turbulent lifted flames. Combust Theory Model 2007;11(2):227-257.

[3] Robin V, Mura A, Champion M, Degardin O, Renou B, Boukhalfa M. Experimental and numerical analysis of stratified turbulent V-shaped flames. Combust Flame 2008;153:288-315.

[4] Mura A, Champion M. Relevance of the Bray number in the small-scale modeling of turbulent premixed flames. Combust Flame 2009;156:729-733.

[5] Eça L, Hoekstra M. Evaluation of numerical error estimation based on grid refinement studies with the method of the manufactured solutions. Comput Fluids 2009;38:1580-1591.

[6] Anderson JD. Computational Fluids Dynamics: the basics with applications. Mac Graw-Hill Ed;1995.

[7] Choi YH, Merkle CL. The application of preconditioning in viscous flows. J Comp Phys 1993;105:207-223.

[8] Najm HN, Wyckoff PS, Knio OM. A semi-implicit numerical scheme for reacting flows. J Comp Phys 1998; 143:381-402.

[9] Cook AW, Riley JJ. Direct numerical simulation of a turbulent reactive plume on a parallel computer. J Comp Phys 1996;129:263-283.

[10] Rauowens P, Vierendeels J, Merci B. A Solution for the odd-even decoupling problem in pressure-correction algorithms for variable density flows. J Comp Phys 2007;227:79-99.

[11] Figueira da Silva LF, Azevedo JLF, Korzenowski H. Unstructured adaptive grid flow simulations of inert and reactive gas mixtures. J Comp Phys 2000;160:522-540.

[12] Turkel E, Radespiel R, Kroll N. Assessment of preconditioning methods for multidimensional aerodynamics. Comput Fluids 1997;26(6):613-634.

[13] Roller S, Munz C. A low Mach number scheme based on multi-scale asymptotics. Computing and Visualization in Science 2000;3(1-2):85-91.

[14] Turkel E. Review of preconditioning methods for fluid dynamics. Applied Num Mathematics 1993;12:257-284.

[15] Darmofal DL, Schmid PJ. The importance of eigenvectors for local preconditioners of the euler equations. J Comp Phys 1996;127(2):346-362.

[16] Ferziger J, Peric M. Computational methods for fluid dynamics 3rd Ed. New York: Springer-Verlag; 2002.

[17] Dourado WMC, Bruel P, Azevedo JLF. A time-accurate pseudo-compressibility approach based on a unstructured hybrid finite volume technique applied to unsteady turbulent premixed flame propagation. Int J Num Met Fluids 2004;44:1063-1091.

[18] Ramshaw JD, O'Rourke PJ, Stein LR. Pressure gradient scaling method for fluid flow with nearly uniform pressure. J Comp Phys 1985;58(3):361-376.

[19] Amsden AA, O'Rourke PJ, Butler TD. KIVA-II A computer program for chemically reactive flows with sprays. KIVA II manual, LA-11560-MS. Los Alamos National labotarories; 1989.

[20] Wang Y, Trouvé A. Artificial acoustic stiffness reduction in fully compressible, direct numerical simulation of combustion. Combust Theory Model 2004;8(3):633-660.

[21] Papageorgakis GC, Assanis DN. Comparison of linear and non-linear RNG-based $K-\varepsilon$ models for incompressible turbulent flows. Num Heat Transfer, Part B 1999;35(1):22.

[22] Chorin AJ. Numerical solution of the Navier-Stokes equations. Mathematics of Computation 1968;22:745-762.

[23] Lima e Silva ALF, Silveira-Neto A, Damasceno JJR. Numerical simulation of two dimensional flows over a circular cylinder using the immersed boundary method. J Comp Phys 2003;189:351-3706.

[24] Chorin AJ, Marsden JE. A mathematical introduction to fluid mechanics, 3rd ed. New York: Springer-Verlag; 1993.

[25] Patankar SV. Numerical heat transfer and fluid Flow, 3rd ed. McGraw-Hill, New York; 1980.

[26] Colella P, Pao K. A projection method for low speed flows. J Comp Phys 1999;149:245-269.

[27] Karki KC, Patankar SV. Pressure based calculation procedure for viscous flows at all speed in arbitrary configurations. AIAA J 1989;27(9):1167-1174. 
[28] Bell JB. AMR for low Mach number reacting flows. LBNL Report LBNL-54351. Proceedings of the Chicago Workshop on Adaptive Mesh Refinement Methods; 2003.

[29] Lessani B, Papalexandris MV. Time-accurate calculation of variable density flows with strong temperature gradients and combustion. J Comp Phys 2006;212(1):218-246.

[30] Knio OM, Najm HN, Wyckoff PS. A Semi-implicit numerical scheme for reacting flow: II. Stiff, operator-split formulation. J Comp Phys 1999;154(2):428-467.

[31] Rider WJ, Kothe DB, Puckett EG, Aleinov ID. Accurate and robust methods for variable density incompressible flows with discontinuities. Barriers and Challenges in Computational Fluid Dynamics. Kluwer Academic Publishers, Boston, 1998;213-230.

[32] Rhie CM, Chow WL. Numerical study of the turbulent flow past an airfoil with trailing edge separation. AIAA J $1983 ; 21: 1525$.

[33] Shen WZ, Michelsen JA, Sorensen NN, Sorensen JN. An improved SIMPLEC method on collocated grids for steady and unsteady flow computations. Num Heat Transfer, Part B 2003;43:221-239.

[34] Versteeg HK, Malalasekera W. An introduction to computational fluid dynamics. Ed. Prentice Hall; 1995.

[35] Harlow FH, Welsh JE. Numerical calculation of time dependent viscous incompressible flow with free surface. Phys Fluids 1965;8:2182-2189.

[36] Ceniceros HD, Roma AM. A nonstiff, adaptive mesh refinement-based method for the Cahn-Hilliard equation. J Comp Phys 2007;255(2):1849-1862.

[37] Ascher UM, Ruuth SJ, Wetton BTR. Implicit-explicit methods for time-dependent partial differential equations. SIAM J Num Analysis 1995;32(3):797-823.

[38] Villar MM. Analise numérica detalhada de escoamentos multifasicos bidimensionais, PhD Thesis. Universidade Federal de Uberlandia, Uberlandia, MG, Brasil; 2007.

[39] Nicoud F. Conservative high-order finite-difference schemes for low Mach number flows. J Comp Phys 2000;255(2):1849-1862.

[40] Bogey C, Bailly C. A family of low dispersive and low dissipative explicit schemes for flow and noise computations. J Comp Phys 2004;194:194-214.

[41] Jameson A, Schmidt W, Turkel E. Numerical solution of the Euler equations by finite volume methods using RungeKutta time stepping schemes. AIAA Fluid and Plasma Dynamics Conference 1981; 15 p.

[42] Schneider GE, Zedan M. A Modified strongly implicit procedure for the numerical solution of field problems. Num Heat Transfer 1981;4:1-19.

[43] Steinberg S, Roache PJ. Symbolic manipulation and computational fluid dynamics. J Comp Phys 1985;57(2):251284.

[44] Roache PJ, Knupp P, Steinberg S, Blaine RL. Experience with benchmark test cases for groundwater flow. ASME FED, Vol. 93, Benchmark Test Cases for Computational Fluid Dynamics, I. Celik and C.J. Freitas, Eds., Book No. H00598-1990 49-56; 1990.

[45] Roache PJ. Verification of codes and calculations. AIAA J 1998;36:696-702.

[46] Salari K, Knupp P. Code verification by the method of manufactured solutions. Sandia report, SAND2000-1444. Sandia National Laboratories; 2000.

[47] Shunn L, Ham F. Method of manufactured solutions applied to variable-density flows solvers. Annual Research Briefs - Center for Turbulence Research 2007;155-168.

[48] Shunn L, Knupp P. Verification of the low-mach number combustion codes using the method of manufactured solutions. CSRI Summer proccedings - Sandia National Laboratories 2007;126-136.

[49] Nos RL. Simulacões de escoamentos tridimensionais bifásicos empregando métodos adaptativos e modelos de campo de fase, PhD Thesis. Universidade de Sao paulo, Sao Paulo, SP, Brasil; 2007.

[50] Ceniceros HD, Nós RL, Roma AM. Three-dimensional, fully adaptive simulations of phase-field fluid models. Unpublished results - paper submitted to J Comp Phys 2009.

[51] Moreau P, Boutier A. Laser velocimeter measurements in a turbulent flame. Symposium (international) on combustion, 1977;16:1747-1756.

[52] Magre P, Moreau P, Collin G, Borghi R, Péalat M. Further Studies by CARS of premixed turbulent combustion in a high velocity Flow. Combust Flame 1988;71:147-168.

[53] Smagorinsky, J. General Circulation Experiments with Primitive Equations. Monthly Weather Review 1963;91:99164.

[54] Klein M, Sadiki A, Janika J. A digital filter based generation of inflow data for spatially developing direct numerical or large eddy simulations. J Comp Phys 2003;186:652-665.

[55] Jeong J. Hussain F. On he identification of a vortex. J Fluid Mech 1995; 285:69-94. 